\title{
Dopamine and Memory: Modulation of the Persistence of Memory for Novel Hippocampal NMDA Receptor-Dependent Paired Associates
}

\author{
Ingrid Bethus, Dorothy Tse, and Richard G. M. Morris \\ Laboratory for Cognitive Neuroscience, Centre for Cognitive and Neural Systems, The University of Edinburgh, Edinburgh EH8 9JZ, United Kingdom
}

\begin{abstract}
Three experiments investigated the role in memory processing of dopamine (DA) afferents to the hippocampus (HPC) that arise from the ventral tegmental area. One hypothesis is that $D_{1} / D_{5}$ receptor activation in HPC is necessary for the encoding of novel, episodic-like information; the other is that DA activation ensures the greater temporal persistence of transient hippocampal memory traces. Rats $(n=$ 35) were trained, in separate experiments using an episodic-like memory task, to learn six paired associates (PAs) in an "event arena" involving a repeated association between specific flavors of food and locations in space. After 6 weeks of training, rats had learned a "schema" such that two new paired associates could be acquired in a single trial in one session (episodic-like memory). We show that encoding of novel PAs is sensitive to intrahippocampal microinfusion of the NMDA antagonist D-AP-5. Experiment 1 established that intrahippocampal infusion of the $\mathrm{D}_{1} / \mathrm{D}_{5}$ dopaminergic antagonist SCH23390 $[R(+)$-7-chloro-8-hydroxy-3-methyl-1-phenyl-2,3,4,5tetrahydro-1 $\mathrm{H}$-3-benzazepine hydrochloride] before encoding of new PAs caused impaired memory $24 \mathrm{~h}$ later but that SCH 23390 had no effect on the later memory of previously established PAs. Experiment 2 established that $\mathrm{SCH} 23390$ modulated the persistence of new memories over time ( $30 \mathrm{~min}$ vs $24 \mathrm{~h}$ ) rather than affecting initial encoding. Experiment 3 revealed that the impact of SCH23390 was not mediated by state dependence nor had an effect on memory retrieval. These findings support the second hypothesis and establish that persistent, long-term memory of rapid, hippocampal-mediated acquisition of new paired associates requires activation of $D_{1} / D_{5}$ receptors in HPC at or around the time of encoding.
\end{abstract}

\section{Introduction}

What function(s) does dopamine serve in the hippocampus (HPC) during learning? The aim of these experiments was to contrast two alternatives: (1) it is critical for encoding, or (2) it is critical for the persistence of memory traces at or around the time of memory encoding. Both possibilities are consistent with the hypothesis of Lisman and Grace (2005) of novelty modulation of hippocampal memory processing.

The dopaminergic mesocorticolimbic pathway arises from the ventral tegmental area (VTA) and is activated by novelty (Steinfels et al., 1983), but the functional impact of this dopamine (DA) neurotransmission may depend on the neural system to which it is afferent. For example, although triggered in common by novelty, its impact in the HPC may differ from the errordetection role that DA serves in the striatum (Schultz et al., 1992; Schultz and Dickinson, 2000). Anatomical, cell-biological, and neuropharmacological data have indicated a VTA projection to the HPC, the presence of DA in the dorsal HPC, and the presence

Received June 9, 2009; revised Nov. 23, 2009; accepted Nov. 25, 2009.

This work was supported by a Medical Research Council programme grant (held by R.G.M.M.) and fellowships from Fondation pour la Recherche Médicale, Fondation Lavoisier, and Singer et Polignac. We are grateful to Patrick A. Spooner and Jane Tulloch for technical assistance and to Roger Redondo and lain Wilson for discussion.

Correspondence should be addressed to Ingrid Bethus, Laboratory for Cognitive Neuroscience, Centre for Cognitive and Neural Systems, The University of Edinburgh, 1 George Square, Edinburgh EH8 9JZ, UK. E-mail: ingrid.bethus@ed.ac.uk.

DOI:10.1523/JNEUROSCI.2721-09.2010

Copyright $\odot 2010$ the authors $\quad 0270-6474 / 10 / 301610-09 \$ 15.00 / 0$ of metabotropic $\mathrm{D}_{1}$ receptors whose activation can lead to transcriptional regulation of plasticity-related genes such as the cAMP response element-binding protein CREB (Dahlström and Fuxe, 1964; Swanson, 1982; Gasbarri et al., 1994, 1997; Lazarov et al., 1998; Jay, 2003). Electrophysiological studies in vivo and in vitro point to a specific role for DA in the temporal persistence of long-term potentiation (LTP) (Frey et al., 1990, 1991; Huang and Kandel, 1995; Swanson-Park et al., 1999; O'Carroll and Morris, 2004).

If the impact of novelty within HPC is, via VTA activation, to extend the "persistence" of synaptic potentiation (Morris, 2006), it follows that a memory task that requires activity-dependent synaptic plasticity within HPC at encoding should also show delay-dependent sensitivity to local $D_{1}$ receptor blockade. Memory traces may be successfully encoded and stored as a spatial distribution of changes in synaptic weights (Martin et al., 2000), but such changes may not persist. Previous work has examined the DA dependence of learning for step-down inhibitory avoidance, the radial maze, and water maze (Packard and White, 1991; Gasbarri et al., 1996; Bernabeu et al., 1997; O'Carroll et al., 2006) but not yet the memory delay dependency of such tasks. We therefore used a new task in which rats learn flavor-place paired associates (PAs) (Day et al., 2003) in a protocol that begins with the "schema" training of six of these PAs across several weeks (Tse et al., 2007). We then introduced novel PAs for a single trial of training and tested memory for these after short or long delays. Using this hippocampal-dependent associative recall paradigm, which en- 
A

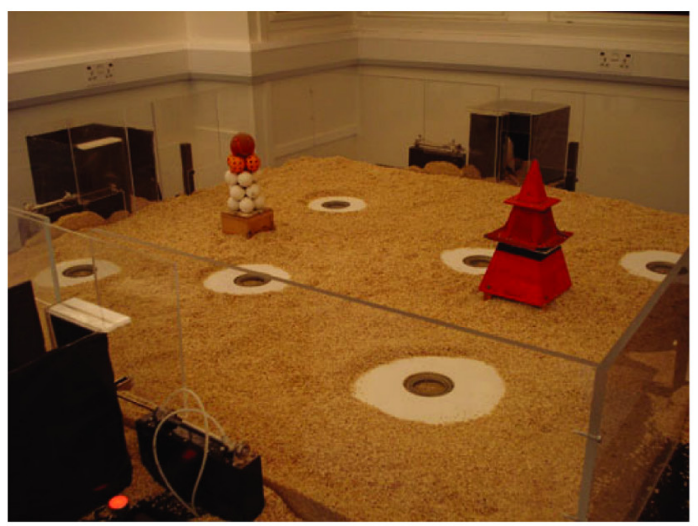

B
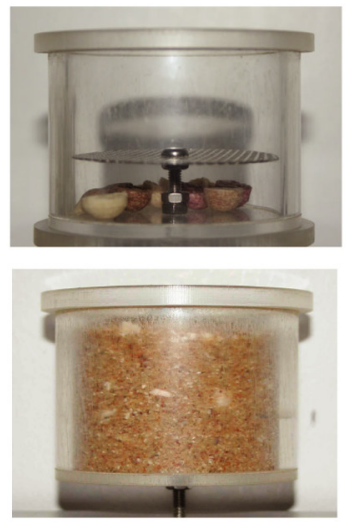

C

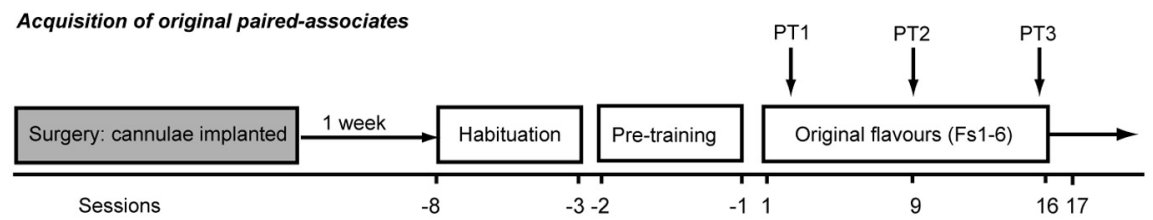

Rapid learning of new paired-associates (SCH23390 $1 \mu \mathrm{g}, 5 \mu \mathrm{g} ; \mathrm{NaCl})$

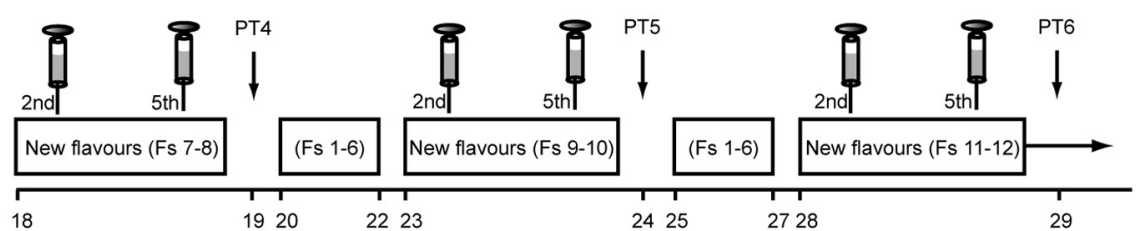

Cued-recall of the original paired-associates ( $\mathrm{SCH} 233901 \mu \mathrm{g} ; \mathrm{NaCl}$ )

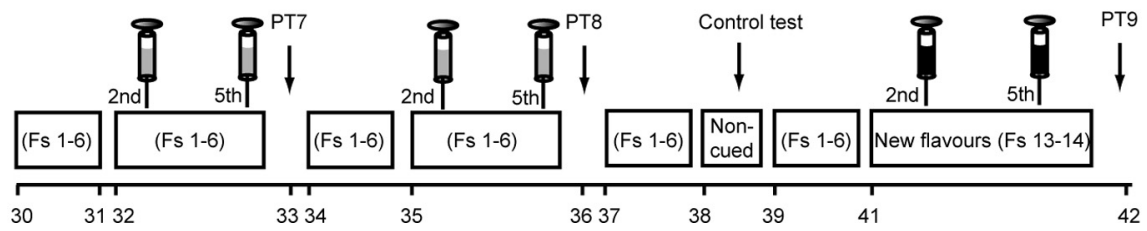

Figure 1. Experimental apparatus and design. $\boldsymbol{A}$, Layout of the event-arena apparatus $(1.6 \times 1.6 \mathrm{~m})$ with the four start boxes and the six sand wells available to which the animals can run to collect food. $\boldsymbol{B}$, Top, Example of an empty sand well with an inner diameter of $6 \mathrm{~cm}$ and a total depth of $5 \mathrm{~cm}$. The sand well contains a grid $3.5 \mathrm{~cm}$ from the top under which $1 \mathrm{~g}$ of each of the six flavors pellets were placed to provide comparable olfactory cues at each sand well. B, Bottom, An example of a "full" sand well with the food pellets hidden, including any accessible reward pellets of a specific flavor. C, Schema of training and new paired associates. Timeline showing the design of experiment 1 (Fs, flavors). The syringes indicate the time of the bilateral iHPC infusions (before the beginning of the second and fifth trials on the training day of the new flavors).

ables rapid learning against the background of a learned schema, we examined the impact of intrahippocampal (iHPC) infusion of the $\mathrm{D}_{1} / \mathrm{D}_{5}$ receptor antagonist $\mathrm{SCH} 23390[R(+)$-7-chloro-8- hydroxy-3methyl-1-phenyl-2,3,4,5-tetrahydro- $1 \mathrm{H}$-3-benzazepine hydrochloride]. We also checked that the recall of memory acquired in the presence of SCH23390 is not state dependent (i.e., only effective if the drug is also present during recall). A novel withinsubjects protocol enabled successive, single-trial encoding experiences of novel PAs across days (episodic-like memory) and thus the possibility of a definitive comparison of the performance of individual subjects in each of three separate experiments.

\section{Materials and Methods}

The detailed methods sufficient for independent replication are described in the supplemental data (available at www.jneurosci.org as sup- plemental material), with information given here sufficient for understanding the training protocols and their rationale.

Animals and surgery. The subjects (experiment $1, n=11$; experiment $2, n=12$; experiment $3, n=12$ ) were group-housed adult male Lister-hooded rats (Charles River), maintained at $85 \%$ of their free-feeding weight. Standard surgical procedures, under isoflurane anesthesia, were used to implant 26 gauge cannulae for drug infusions targeted at the dorsal hippocampus bilaterally. The rats had a $7 \mathrm{~d}$ recovery period before the start of behavioral procedures.

Apparatus. The "event arena" (Fig. 1A) was made of clear Plexiglas $(1.6 \times 1.6 \mathrm{~m})$, with the floor containing a $7 \times 7$ grid of 49 circular holes $(6 \mathrm{~cm}$ diameter, $20 \mathrm{~cm}$ spacing, with plastic lids level to the floor) and covered by $3 \mathrm{~cm}$ of sawdust. Two distinctive intra-arena landmarks, a glued stack of golf balls and a pyramid, were placed in two locations: row 4 , column 2 and row 4 , column 6 of the $7 \times 7$ grid. Access to the arena was from any of four Plexiglas start boxes $(25 \times 25 \times 25 \mathrm{~cm})$, covered with black paper to make them dark inside, that were located centrally in each sidewall. These had computer-controlled sliding doors for arena access. Plastic sand wells could be inserted into the circular holes in the floors of the arena, and food rewards $(0.5 \mathrm{~g}$ pellets manufactured in various flavors, all with equal nutritional value) could be placed inside them (Fig. $1 B$ ). The sand was mixed with ground-up food pellets of all flavors used in an 90:10 ratio. A key design feature of these sand wells was a false floor with a lower compartment containing masking odors and flavors (Fig. $1 B$, contrast top and bottom pictures), these being obscured by the added sand mixture.

Drugs and drug infusions. The $\mathrm{D}_{1} / \mathrm{D}_{5}$ antagonist SCH23390 was used at concentrations of $5 \mu \mathrm{g} / \mu \mathrm{l}(15.4 \mathrm{~mm})$ and $1 \mu \mathrm{g} / \mu \mathrm{l}(3 \mathrm{~mm})$ and was made up as described in supplemental Methods (available at www.jneurosci.org as supplemental material). The competitive NMDA antagonist D-AP-5 was used at a concentration of $5.9 \mu \mathrm{g} / \mu \mathrm{l}(30 \mathrm{~mm})$. The $\mathrm{pH}$ of the drug solutions was adjusted to 7.2 by the addition of 1 $\mathrm{M} \mathrm{NaOH}$ solution. Standard procedures were used for intracerebral drug infusions at a rate of $0.2 \mu \mathrm{l} / \mathrm{min}$ over $5 \mathrm{~min}$, after which the infusion cannulae were left in place for an additional 2 min to avoid backflow and stylets were re-

placed into the guide cannulae.

Behavioral training. Within-subjects, repeated-measures designs were used in three experiments that each consisted of a series of initial training sessions (Fig. 1C). The original arrangement of flavors (F1-F6) is shown in supplemental Figure S1, $A$ and $B$ (available at www.jneurosci.org as supplemental material). New PAs (F7-F14) (supplemental Fig. S1A, available at www.jneurosci.org as supplemental material) were trained later, with and without drug infusions, followed by interposed memory probe tests (PTs). The primary focus of these additional tests was to examine the impact of blockade of hippocampal $D_{1} / D_{5}$ receptors on short-term and long-term (LTM) memory and state dependence. We also scheduled various essential control conditions. Sessions were numbered from the first paired-associate training session (experiment 1) (Fig. $1 C$ ). During the main part of each experiment, the animals were trained on alternate days in two cohorts of up to six rats with each cohort receiv- 
ing three training sessions per week (cohort 1: Monday, Wednesday, and Friday; cohort 2: Tuesday, Thursday, and Saturday).

The initial training sessions consisted of the following: (1) habituation (sessions $-8-3$ ); (2) acquisition of original schema of six flavor paired associates (sessions 1-17); and (3) nonrewarded PTs (sessions 2, 9, 16).

For performance measures, several parameters were measured during training trials: (1) the number of incorrect sand wells at which digging occurred before choosing the correct sand well [primary error measure: chance, 2.5 errors; conversion to a performance index $=100-100$ * (errors/5)]; (2) latency to dig at the correct sand well (seconds); (3) a "choice" was recorded only when a rat placed its front paw on or into a sand well. Rats running past or sniffing around one were not considered as making a choice, because running around a nonchosen well was an inevitable feature of the arena design. In rare cases, it was difficult to tell from the video monitors whether or not the rats had made a choice as so defined. In this case, when the experimenters entered the room at the end of a trial, they checked carefully whether there were any traces of digging, i.e., whether the sand had been displaced around the sand well(s).

To calculate the primary measure of memory recall, in probe tests, the time spent digging at each of the six sand wells over the 120 s test (none of which contained reward during a probe test). The primary measure of memory recall, calculated as the proportion of time spent at the cued location relative to the average time at the noncued locations, including the noncued location of the other of each of two novel flavors introduced during a training day. Although not rewarded during the $120 \mathrm{~s}$ probe test time, the rats were given three half pellets (correct flavor) in the correct location at the end of probe tests to limit extinction.

Experiment 1: does $D_{1} / D_{5}$ receptor activation in HPC contribute to the memory of novel paired associates? The primary focus of experiment 1 was to investigate the impact of blocking $\mathrm{D}_{1}$ receptors in the hippocampus on new and old paired associates. After this, we conducted an important control test and checked the hippocampal dependence of the learning of new paired associates by investigating its sensitivity to intrahippocampal NMDA receptor blockade: (1) training and probe tests (Fig. 1C, sessions 18-29), impact of SCH23390 on new learning; (2) training and probe tests (Fig. 1C, sessions 30-36), impact of SCH23390 on previously trained flavors; (3) noncued control test; and (4) training and probe tests (Fig. 1C, sessions 41-42), impact of D-AP-5 during learning.

Experiment 2: does $D_{1} / D_{5}$ receptor activation play a role in memory encoding or the long-term persistence of novel paired associates? The purpose of the second experiment was to investigate the effect of blocking $D_{1} / D_{5}$ receptors in HPC on the short-term or long-term memory of the new paired associates. The aim was to establish whether SCH23390 affected memory encoding or the temporal persistence of newly formed memories. Sessions 25 onward focused on short-term versus long-term memory (design in supplemental Fig. S2, available at www.jneurosci.org as supplemental material). A new batch of animals was used $(n=12)$.

Experiment 3: can the impact of $a D_{1} / D_{5}$ antagonist memory be explained in terms of state dependency, memory retrieval, or the persistence of newly encoded information? The aim of the third experiment was to test whether (1) the results of experiment 2 could be interpreted in terms of state dependency and (2) whether intra-HPC SCH23390 affects memory retrieval. State-dependent learning is a phenomenon in which information learned in one state of the organism is retrieved best if a similar state is reinstated at the time of the testing (Dudai, 2002). Sessions 18 onward focused on specific manipulations to investigate state dependency and retrieval (design in Fig. S3, available at www.jneurosci.org as supplemental material). A new batch of animals was trained $(n=12)$.

Perfusion and histology. All rats were terminally anesthetized with Euthatal (Rhône Mérieux) and then perfused intracardially with $0.9 \%$ saline, followed by $4 \%$ Formalin. The brains were removed and stored in $4 \%$ Formalin for a minimum of $24 \mathrm{~h}$. Coronal $30 \mu \mathrm{m}$ sections were cut using a cryostat with one in every five sections recovered for histological analysis. These sections were mounted on slides, stained with cresyl violet, and coverslipped using DPX (mixture of distyrene, tricresyl phosphate, and xylene). The sections were examined with a light microscope under 20-fold magnification to verify cannulae placements. For each brain, the infusion site was plotted by determining the deepest point at which tissue damage was evident and marking this location on the ap- propriate coronal section taken from the atlas of Paxinos and Watson (1998) (see Fig. 3C).

Statistical analyses. All numerical data are presented as mean \pm SEM. For all experiments, the number of errors was assessed in the main training sessions and time spent digging in each sand well during the probe tests. The latency to dig at the correct well was also routinely analyzed, but these data never showed significant differences but are presented for experiment 1 only in the supplemental data (available at www.jneurosci. org as supplemental material). Statistical significance was determined by repeated-measures ANOVAs and, when appropriate, one-sample $t$ tests. The Greenhouse-Geisser correction for repeated measures was applied (with appropriate alteration of degrees of freedom). In experiments 1 and 2, after the ANOVA, pairwise comparisons were conducted with a Bonferroni's correction for multiple comparisons. In experiment 3 , a set of appropriate orthogonal comparisons was created to analyze the impact of SCH23390 on persistence, state dependency, and/or memory retrieval.

\section{Results}

Before turning to the main results, it is important to convey qualitatively how event-arena experiments of this kind proceed. After habituation and pretraining, the animals readily eat the "cue" food in the start box and then, after exploratory head movements at the entrance to the arena (Johnson and Redish, 2007), enter it and run between the sand wells in search of the available reward of the same flavor. They dig through the sand at these wells and, at the correct one, soon find the $0.5 \mathrm{~g}$ food pellets that they then carry (one by one) back to the start boxes to eat. This natural carrying behavior is typical of rats (Maaswinkel and Whishaw, 1999), and we made no attempt to prevent it; in fact, unpublished observations in our laboratory indicate that having the animals eat the retrieved food in the start box adds to their ability to use this flavor as a recall cue on later sessions, presumably because this cue is given at a start-box location.

\section{Experiment 1: gradual acquisition of paired-associate memory, a schema, and then rapid drug-dependent acquisition of new paired associates (SCH23390 and D-AP-5)} As shown in Figure $2 A$, the animals are initially at chance on the baseline task, but they improve across 15 sessions to a performance index (PI) score of between 70 and $80 \%$. This is lower than the 90 or $95 \%$ demanded in standard discrimination-learning experiments; however, these typically consist of only two choices, whereas there are six choices here. The $z$-scores for the PI relative to chance were, at asymptote, around or greater than $z=5$, and this is indicative of highly significant choice performance. Once this level is reached, the main part of each study commences with the within-subjects introduction of new PAs in the presence or absence of drugs.

After the training phase (Fig. 2 A, sessions 1-17, white background) in which the animals were trained on alternate days to learn concurrently six paired associates (each flavor associated to a distinct location; see supplemental data, available at www. jneurosci.org as supplemental material), we tested the effect of $\mathrm{SCH} 23390$ on (1) the rapid acquisition of the new PAs (intraHPC infusions given bilaterally 20 min before new PAs exposure) and (2) the memory of the previously acquired six PAs.

During the training phase, three initial nonrewarded probe tests (PT1-PT3) were scheduled as shown in Figure $1 C$ (top row) to examine acquisition of paired-associate memory. Figure $2 B$ represents percentage of dig time at the cued location (black bars) relative to that at the noncued locations (white bars). These probe tests revealed, as expected, a graded learning of the original PAs from sessions $1-16(F=23.16, \mathrm{df}=1.75 / 17.51, p<0.001) . t$ tests 

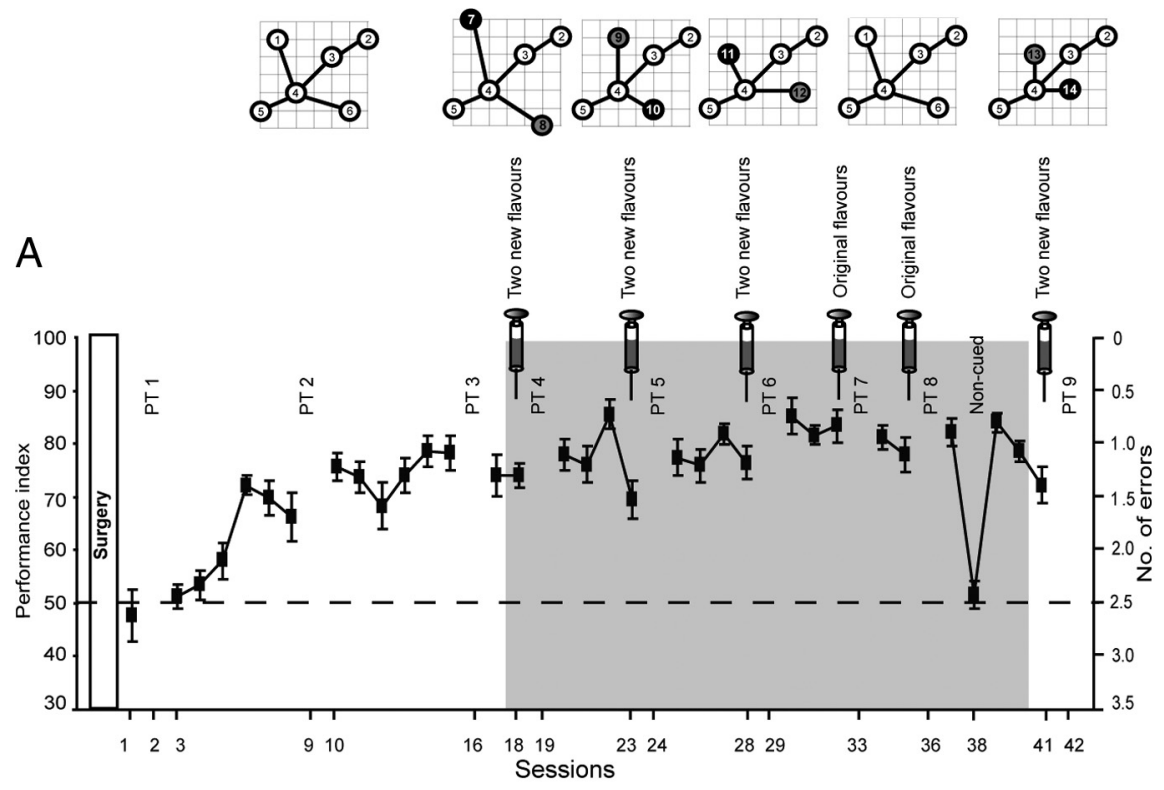

B

C
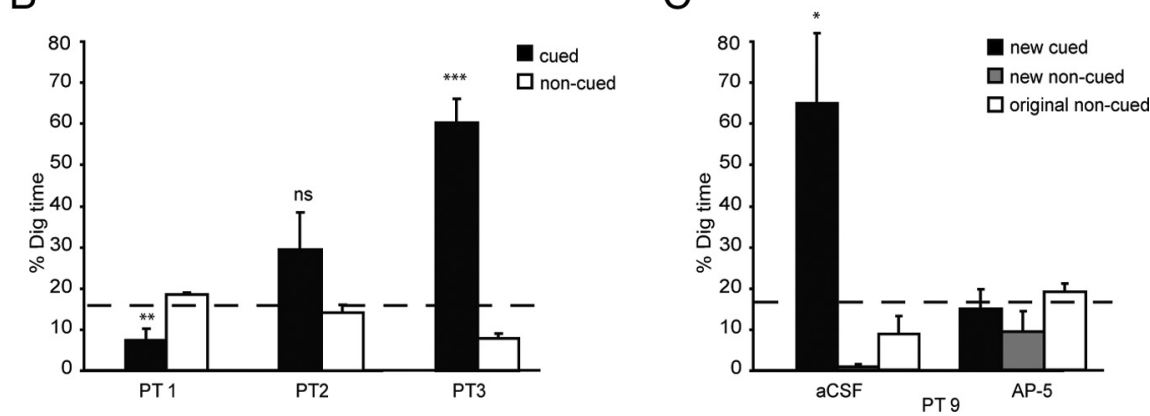

Figure 2. Acquisition of the schema, new paired associates, and NMDA dependency. $\boldsymbol{A}$, Performance index of acquisition of the original six paired associates (sessions 1-17; white background). Single days of training of new paired associates alternated with $3 \mathrm{~d}$ of the original schema (sessions $18-42$; gray background). Performance rose to a stable level of $80 \%$ with minimal variability. Note that performance fell to chance on session 38 when the start-box flavor cues were absent. $\boldsymbol{B}$, Three cued-recall probe trials for the acquisition of the original schema. The graph represents percentage of dig time at the cued location (black bars) relative to that at the noncued locations (white bars). C, Hippocampal NMDA receptor dependence of the acquisition of the new paired associates. The graph represents percentage dig time at the new cued location (black bars) and the new noncued location (gray bars), as well as the average of the four original paired associates (white bars) for probe test 9 in the control (aCSF) and drug (AP-5) conditions. ns, Nonsignificant. ${ }^{*} p<0.05 ;{ }^{* *} p<0.01 ;{ }^{* *} p<0.001$ for $t$ tests comparing the proportion of digging for the cued paired associates relative to chance level of $16.7 \%$.

comparing the proportion of digging for the cued PA were above chance in PT3 $(t=7.76, \mathrm{df}=10, p<0.001)$.

At the end of the study (sessions 41-42), we sought to extend our finding of the hippocampal dependence of PA acquisition that had been established using lesions (Tse et al., 2007). A next step was to examine the possible role of hippocampal plasticity, via $\mathrm{HHPC}$ infusion of the NMDA antagonist D-AP-5 at the time of novel PA encoding. Because the primary focus of this experiment was on the impact of SCH23390 on the memory of novel and familiar PAs, collection of the D-AP-5 data was not until PT9 (Fig. 1C, bottom row). Figure $2 C$ presents dig time data of this probe test in session 42. Because the drug was given on session 41, $20 \mathrm{~min}$ before the trials for each of two new PAs that were trained in one session, the graph includes the relative digging during the probe test $24 \mathrm{~h}$ later at both the new cued PA and the new noncued PA, as well as the average of the four original PAs. Of the 10 animals that completed training to this stage of the study (from an initial $n=11$ ), half were give D-AP-5 and the others vehicle. The ANOVA revealed an interaction between drug (a between-

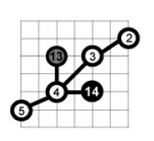

subjects factor $)$ and paired associates $(F=$ 10.04 , df $=1.14 / 9.15, p<0.01)$. The pairwise comparisons analysis with Bonferroni's corrections for multiple comparisons showed a significant difference between the control group [artificial CSF (aCSF)] and the D-AP-5 group only on digging at the location of new cued paired associates (Fig. $2 C$, black bars $)(p<0.05)$. Additional $t$ tests compared the proportion of digging for the new cued PA relative to chance, revealing that digging was above chance for the control group (aCSF, $t=$ $3.14, p<0.035)$ but not for the D-AP-5 group (D-AP-5, $t<1$, NS). This finding confirms the HPC dependence of novel PA acquisition and indicates that it involves local NMDA receptor activation.

Revealing the hippocampal dependency of the task sets the stage for a focused analysis of the impact of iHPC SCH23390 on memory for PAs. Figure 3 shows a decline in memory at $24 \mathrm{~h}$ associated with SCH23390 present at new encoding.

SCH23390 and new encoding

The ANOVA revealed an interaction between drug and digging locations $(F=$ 8.5, df $=2.49 / 24.9, p<0.001)($ Fig. $3 A$ ). Pairwise comparisons, with Bonferroni's corrections for multiple comparisons and focusing exclusively on digging at the new cued location across drug conditions (i.e., black bars), showed significantly less digging when the animals were treated with SCH23390 ( $1 \mu \mathrm{g}, p<0.05 ; 5$ $\mu \mathrm{g}, p<0.01)$ than with saline. Similarly, $t$ tests that compared the proportion of digging at the cued location indicated above chance performance in the control condition $(t=3.42, \mathrm{df}=10, p<0.01)$ but not for the SCH22390 $1 \mu \mathrm{g}$ condition $(t=$ 1.02 , df $=10$, NS). Unexpectedly, there was less digging than expected by chance in the SCH23390 $5 \mu$ g condition $(t=9.80$, df $10, p<0.05)$. Given this, and considering that our lower concentration of SCH 23390 was sufficient to impair memory at $24 \mathrm{~h}$, we used only the lower concentration in later experiments.

\section{SCH23390 and previously trained memories}

Figure $3 B$ shows the lack of an effect of SCH23390 on memory of previously trained PAs. An overall ANOVA revealed a difference in dig time at the cued and noncued locations $(F=44.80, \mathrm{df}=$ $1 / 10, p<0.001)$ but no significant interaction between drug and cued location $(F<1)$. $t$ tests comparing the proportion of digging for the cued PA relative to chance were significant for both conditions $(\mathrm{NaCl}: t=4.12, \mathrm{df}=10, p<0.002$; SCH23390 at $1 \mu \mathrm{g}: t=$ 9.88, $\mathrm{df}=10, p<0.001)$.

It should be noted that the mean time spent digging at the cued location for the original trained PAs (57.5 s in Fig. $3 B$ ) was higher than that for the new PAs in the $\mathrm{NaCl}$ group ( $43.5 \mathrm{~s}$ in Fig. $3 A$ ). This difference is to be expected given that the original PAs had, by this point, 21 training trials (sessions 1-32), whereas new 
A
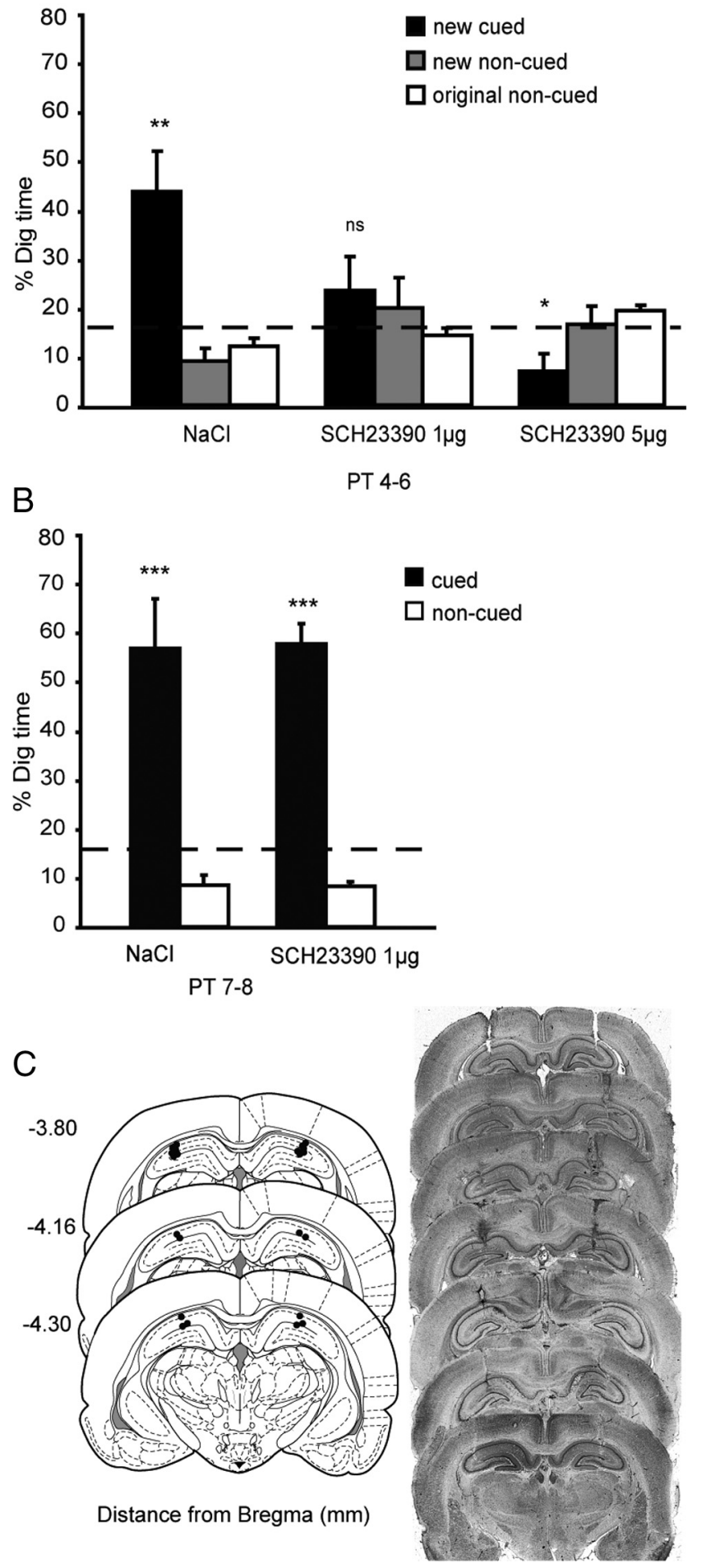

Figure 3. Experiment 1: $\boldsymbol{A}$, Dopamine dependency of encoding of new paired associates. Percentage dig time in all three conditions ( $\mathrm{NaCl}, \mathrm{SCH} 233901 \mu \mathrm{g}$, and SCH23390 5 $\mu \mathrm{g})$ across the three counterbalanced probe tests (PT4-PT6). B, SCH23390 and previously trained memories. Percentage dig time for the original paired associates in two conditions ( $\mathrm{NaCl}$ and SCH23390 $1 \mu \mathrm{g}$ ). C, The left shows plots of the locations of cannulae tips ( $n=11$ per HPC; experiment 1$)$. Infusion sites are marked on the appropriate section of a stereotaxic brain atlas (Paxinos and Watson, 1998). The right shows Niss--stained sections showing examples of representative cannulae tracks in the dorsal hippocampus in each hemisphere of the brain. ns, Nonsignificant. ${ }^{*} p<0.05$; ${ }^{* *} p<0.01 ;{ }^{* * *} p<0.001$.
PAs only ever have a single training trial. Nonetheless, it is striking that the probe test score for a new PA trained in the presence of saline is as good as it is and well above chance. That is, against the background of a trained schema, new PA learning can occur in one trial.

Latency to dig at correct sand well on the day of drug infusions We also examined whether SCH23390 had any impact on the latency with which the animals completed a trial (on sessions 18, 23, 28 and sessions 32, 35). On the first three of these, we could make a within-session comparison of new PA trials (in the presence or absence of the drug) with the latency on the remaining original PA trials without the drug. Latencies on the new PA trials averaged $38.5 \mathrm{~s}$ and varied between 15 and $50 \mathrm{~s}$ depending, in part, on the proximity of the sand well to the start box and the number of errors made (supplemental Fig. S4A, available at www.jneurosci.org as supplemental material). An ANOVA of these latency scores revealed no overall interaction between drug and trial $(F=2.5, \mathrm{df}=1.72 / 17.2, p>0.1)$, although the graph showed a nonsignificant increase in latency for new PA trials in the presence of SCH23390. These data are discussed in the supplemental data (available at www.jneurosci.org as supplemental material). In the last two sessions examined (sessions 32 and 35), we could make a within-session comparison of the impact of the drug on the latency to complete original PA trials (supplemental Fig. S4 B, available at www.jneurosci.org as supplemental material). The animals were typically faster, taking between 15 and $22 \mathrm{~s}$ to complete a trial, with the ANOVA also revealing no interaction between drug and trial $(F=3.8, \mathrm{df} 1 / 10, p>0.05)$. These findings indicate that, $20 \mathrm{~min}$ after an SCH23390 injection, there was no significant locomotor impairment that might have affected memory encoding in an indirect manner.

\section{Cannula placement}

Inspection of the location of the tips of the cannulae in coronal sections of the brain of all tested animals revealed these to be located in dorsal hippocampus, in the CA1 region of the HPC (Fig. 3C).

Experiment 2: $D_{1} / D_{5}$ receptors are critical for the memory persistence but not the encoding of new paired associates The aim of experiment 2 was to compare the impact of SCH 23390 on short- and long-term memory. To test this hypothesis, a new batch of animals $(n=12)$ were trained to learn the six pairedassociates schema, followed by the introduction and training new PAs preceded 20 min before by intra-HPC drug or vehicle infusions. The memory probe tests proceeded after a short (30 min) or a long (24 h) memory delay.

Impact on short-term versus long-term memory

Figure 4 shows the percentage dig time in the series of four counterbalanced probe tests conducted $30 \mathrm{~min}$ or $24 \mathrm{~h}$ after the encoding of new paired associates. An overall ANOVA revealed a triple interaction between memory delay, drug, and paired associate $(F=18.16, \mathrm{df}=1.94 / 21.38, p<0.001)$. Pairwise comparisons, again with Bonferroni's corrections for multiple comparisons, focused on digging at the new cued paired associates (black bars). This showed no significant difference between the $\mathrm{NaCl}$ and $\mathrm{SCH} 22390$ conditions at a 30 min delay $(F=2.1$, $p>0.18)$ but a significant decline in memory in the SCH23390 condition at $24 \mathrm{~h}(F=5.6, p<0.04)$. Separate comparisons across time intervals revealed no difference between the two probe tests at $30 \mathrm{~min}$ and $24 \mathrm{~h}$ with $\mathrm{NaCl}(F<1)$ but a highly significant delay-dependent decrease across the two SCH23390 


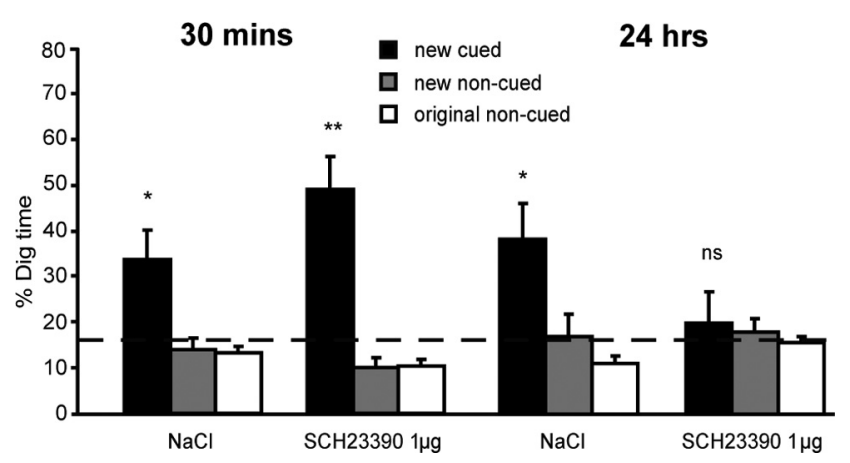

Figure 4. Experiment 2: delay dependency. Impact of the $\mathrm{SCH} 23390$ on short-term (30 $\mathrm{min}$ ) and long-term ( $24 \mathrm{~h}$ ) memory. Percentage dig time at both delays ( $30 \mathrm{~min}$ or $24 \mathrm{~h}$ ) in two drug conditions. Note good performance in the presence of the drug at $30 \mathrm{~min}$ but not at $24 \mathrm{~h}$. ns, Nonsignificant. ${ }^{*} p<0.05$; $^{* *} p<0.01$.

tests $(F=16.9, p<0.002)$. Additional $t$ tests compared the proportion of digging for the cued paired associates relative to chance. As shown in Figure 4, this revealed that digging was above chance at $30 \mathrm{~min}$ in both conditions ( $\mathrm{NaCl}: t=2.67, p<0.025$; SCH23390: $t=4.78, p<0.001)$ but only for the $\mathrm{NaCl}$ condition at $24 \mathrm{~h}$ ( NaCl: $t=2.85, p<0.02$; SCH23390: $t=0.5$, NS). Thus, these data reveal a delay-dependent effect of a $\mathrm{D}_{1} / \mathrm{D}_{5}$ antagonist on the memory of novel PAs. These receptors must be activated at encoding for memory to persist.

Experiment 3: the state dependency hypothesis does not explain results obtained in experiments 1 and 2 , and $D_{1} / D_{5}$ receptor blockade in HPC does not affect retrieval

The aim of experiment 3 was to check that the apparent delay dependency of experiment 2 is not an artifact or consequence of state dependency. The phenomenon of "state-dependent" memory, in which information learned in one state (e.g., presence of a drug) is retrieved best if a similar state is reinstated at the time of testing, could explain the apparent impairment of memory of new PAs at $24 \mathrm{~h}$ but not 30 min with SCH23390 because testing of the former but not the latter is in a different drug "state."

This possibility can be tested by performing memory retrieval probe tests similar or different drug conditions to those of exposure to new PAs. A full $2 \times 2$ design was deployed, enabling both an examination of an impact of the drug on memory retrieval and a replication of our findings with respect to memory persistence.

Figure 5 presents the percentage dig time across the four counterbalanced probe tests (PT4-PT7). The ANOVA comparing performance across drug conditions (SCH22390-SCH23390, $\mathrm{NaCl}-\mathrm{SCH} 23390, \mathrm{SCH} 23390-\mathrm{NaCl}$, and $\mathrm{NaCl}-\mathrm{NaCl}$ ) and all dig locations revealed an interaction $(F=5.42$, df $=2.93$ / 32.26, $p<0.005)$.

Inspection of the data suggests that memory for new PAs trained under $\mathrm{NaCl}$ are above chance at retrieval without regard to drug condition, whereas new cued PAs trained in the presence of SCH23390 are at chance. A second ANOVA, focusing on the new cued locations only (Fig. 5, black bars), also showed an interaction between drug and digging location $(F=5.93$, df $=$ 2.1/23.37, $p<0.01)$. This overall $F$ value was partialed out using a set of three orthogonal comparisons that compare groups on the basis of a memory persistence, a state dependency, or a retrieval hypothesis of the impact of SCH23390. The first orthogonal comparison (persistence hypothesis) revealed a significant difference between drug conditions $(\mathrm{NaCl}-\mathrm{NaCl}+\mathrm{NaCl}-$

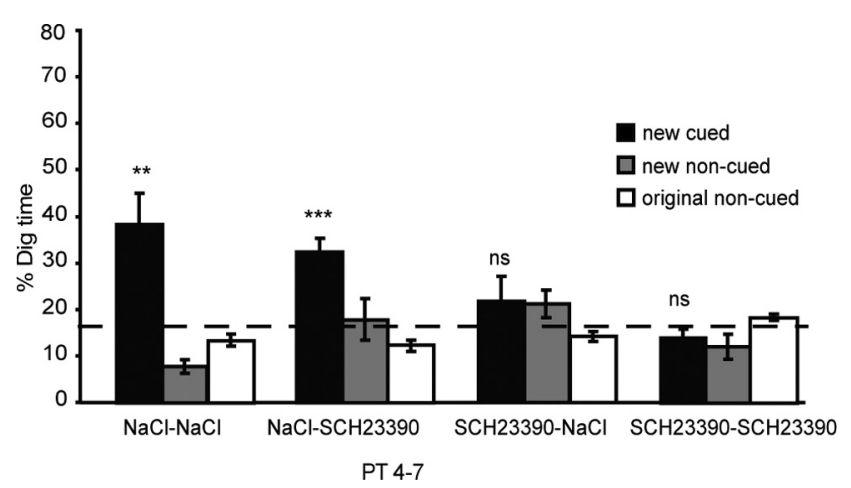

Figure 5. Experiment 3: state dependency and memory retrieval. Percentage dig time across the four counterbalanced probe tests (PT4 -PT7) in all four conditions ( $\mathrm{NaCl}$ before encoding and before retrieval, $\mathrm{NaCl}$ before encoding and SCH23390 before retrieval, SCH23390 before encoding and $\mathrm{NaCl}$ before retrieval, and SCH23390 before encoding and retrieval). Note that memory was impaired only when the drug was present at the time of encoding. ns, Nonsignificant. ${ }^{* *} p<0.01 ;{ }^{* * *} p<0.001$.

$\mathrm{SCH} 23390$ vs SCH $23390-\mathrm{NaCl}+\mathrm{SCH} 23390-\mathrm{SCH} 23390 ; F=$ 10.9 , $\mathrm{df}=1 / 23.4, p<0.005)$, indicating that the drug impaired the memory persistence of new PAs if present at the time of encoding. The state-dependency hypothesis $\mathrm{H} 2(\mathrm{NaCl}-\mathrm{NaCl}+$ SCH23390-SCH23390 vs NaCl-SCH23390 + SCH23390$\mathrm{NaCl})$ was not significant $(F<1)$, and, similarly, the retrieval hypothesis was also nonsignificant $(F=1.59$, NS). Additional $t$ tests compared the time of digging at the new cued PA relative to chance, revealing that digging was above chance in the probe test for drug conditions $\mathrm{NaCl}-\mathrm{NaCl}(t=3.27, p<0.007)$ and $\mathrm{NaCl}-$ SCH23390 $(t=5.69, p<0.001)$ but not for drug conditions $\mathrm{SCH} 23390-\mathrm{NaCl}(t<1$, NS) or SCH23390-SCH23390 $(t=$ $1.37, \mathrm{NS})$.

\section{Discussion}

The main findings are as follows. (1) Intrahippocampal infusion of the $\mathrm{D}_{1} / \mathrm{D}_{5}$ antagonist SCH23390 impaired memory for new PAs in an episodic-like memory task in which rats had previously acquired a flavor-place schema; the drug had no effect on the memory of previously established PAs. (2) SCH23390 modulated the persistence of new memories over time rather than affecting encoding per se. (3) This impact of SCH23390 was neither mediated by state dependence nor affected retrieval. We also established (4) the hippocampal dependency of this cued-recall task using intrahippocampal infusions of the selective NMDA receptor antagonist D-AP-5. These findings have implications for the role of $\mathrm{D}_{1} / \mathrm{D}_{5}$ receptors in $\mathrm{HPC}$ on the neuromodulation of memory processing.

\section{The hippocampal dependence of the PA task}

Our use of this relatively new paired-associate schema task makes it possible, for the first time, to examine the neurobiology of one-trial PA encoding of new information on a within-subjects basis. The task is sensitive to hippocampal lesions (Tse et al., 2007), and our new data establish further that bilateral iHPC infusions of D-AP-5 before the encoding of new PAs results in memory loss when tested $24 \mathrm{~h}$ later. Previous autoradiographic work has established that drug diffusion with this iHPC protocol is primarily restricted to the dorsal HPC (Steele and Morris, 1999). Thus, the task is not only "hippocampal dependent" in the classical sense of being impaired by lesions but is also dependent on NMDA receptor-dependent mechanisms within HPC at the 
time of encoding, presumably involving associative synaptic potentiation (Bliss and Collingridge, 1993). In passing, the previous lesion study was subject to the criticism that ibotenic acid lesions might result in aberrant neocortical activity after the lesion (Rudy and Sutherland, 2008; Tse et al., 2008). The present data renders this concern even less likely because iHPC infusion of D-AP-5 is not known to have such an effect on neocortical activity. Thus, although the memory traces of multiple PAs are probably in neocortex (Bontempi et al., 1999; Maviel et al., 2004; Tse et al., 2007), the encoding of new PAs critically involves an NMDA receptordependent mechanism in HPC, followed by cellular and then rapid systems consolidation.

\section{Functional role of $D_{1} / D_{5}$ receptors in the dorsal hippocampus}

The key new finding is that activation of $\mathrm{D}_{1} / \mathrm{D}_{5}$ receptors in $\mathrm{HPC}$ is critical for the persistence of memory that depends on hippocampal processing at or around the time of encoding. Our new results point to a delay-dependent effect of blocking $D_{1} / D_{5}$ receptors on LTM.

A possible alternative account of the apparent delaydependent memory impairment could be that of state dependency. According to this, poor memory at $24 \mathrm{~h}$ might be explained by the animal being in a different physiological state during memory retrieval in the absence of the drug than it had been at encoding in its presence (Overton, 1964). Good memory at $30 \mathrm{~min}$ could be because the animals are still under the effects of the drug. Experiment 3 tested this by comparing SCH23390 in three conditions: before encoding, before retrieval ( $24 \mathrm{~h}$ later), or before both encoding and retrieval. The results are inconsistent with state dependency because the animals had a similar impairment of memory when SCH23390 was infused only before encoding as when infused before both encoding and retrieval. They also exclude a potential role of $\mathrm{HPC} \mathrm{D}_{1} / \mathrm{D}_{5}$ receptors at or around the time of retrieval, because their iHPC blockade 20 min before the recall test had no effect.

Although we cannot exclude the possibility that SCH23390 has agonist actions at $5-\mathrm{HT}_{2 \mathrm{~A}}$ and $5-\mathrm{HT}_{2 \mathrm{C}}$ receptors at the concentration used (Millan et al., 2001), such actions should have resulted in a facilitation of hippocampus-dependent memory rather than an impairment (Buhot et al., 2000; Harvey, 2003). In addition, the lack of memory impairment in the recall test $30 \mathrm{~min}$ after encoding (50 min after drug infusion) and the absence of difference in latency to dig in the correct sand well between drug and nondrug trials rules out interpretations of our results in terms of hypokinesia (Fink and Smith, 1980; Taghzouti et al., 1985), a reduction of motivation, or an impairment of motorskill learning, such as pellet retrieval (Smith-Roe and Kelley, 2000; Willuhn and Steiner, 2006).

After a long time during which many studies have focused on the role of DA in error-correcting reward processing in the striatum (Schultz, 2007), attention is now being focused on the role of mesohippocampal DA projections to HPC (Lisman and Grace, 2005). Previous studies have implicated DA in both learning and memory by blocking or activating hippocampal DA receptors before (Umegaki et al., 2001) or immediately after (Packard and White, 1991) training or via lesions of mesohippocampal DA projections before training (Gasbarri et al., 1996). However, these studies did not contrast encoding versus persistence. In keeping with the LTP data described in Introduction, O'Carroll et al. (2006) observed an impairment of the persistence of water-maze spatial memory with intra-HPC infusion of SCH23390 at the time of encoding that the present data support.
Recently, an elegant series of experiments (Rossato et al., 2009) has shown a contribution of DA to the maintenance of LTM storage through a mechanism involving $\mathrm{D}_{1}$ receptor signaling in dorsal CA1 that occurs quite a long time (12 h) after acquisition. Previous findings of Bernabeu et al. (1997) are consistent with this new idea about DA involvement in memory consolidation, as is recent work by McNaughton's group (Valdes et al., 2008) who have shown that VTA cells can fire during periods of memory "replay." The possibility that there are actions of DA on memory consolidation at or around the time of encoding (our findings) and separately at later time points are not mutually exclusive. Indeed, the synaptic tagging and capture hypothesis of protein synthesis LTP (Frey and Morris, 1997, 1998) raises the possibility of novelty-associated upregulation of plasticity proteins at a time separate from encoding. These could help stabilize learning-associated synaptic change provided local synaptic tags are set at the time of learning. Data consistent with a role for such a mechanism in memory consolidation have been reported by Moncada and Viola (2007).

\section{Toward better "episodic-like" memory tasks and the impact of novelty on memory persistence}

We used a new PA learning task in which, in the same paradigm and with the same animals, the relative impact of a drug on well trained PAs across days can be distinguished from that on the encoding, storage, and/or retrieval of new PAs. These dissociations are not possible using inhibitory avoidance (Rossato et al., 2009) nor using the free-recall delayed matching-to-place watermaze task (O'Carroll et al., 2006) because, in the latter case, a change in the location of the escape platform entails both acquisition of a new location and extinction of a previous location. The use here of distinct recall cues (different foods) supersedes this limitation. Our new paradigm also confirms that new PAs can be integrated into a preexisting schema after a single training trial (Tse et al., 2007). Such encoding is "episodic-like" in that encoding of both object information (what food) and place information (where in the arena) occurs in a single trial, but it falls short of a "pure" definition of episodic-like memory that involves the triad of "what-where-when," i.e., memory of time as well (Clayton and Dickinson, 1998).

Some recent human imaging studies have also shown that midbrain DA neurons may also play a critical role in modulating HPC-dependent episodic memory (Lisman and Otmakhova, 2001; Schott et al., 2004; Wittmann et al., 2005; Adcock et al., 2006; Shohamy and Wagner, 2008). Shohamy and Wagner (2008) suggest that an HPC-midbrain network may not only provide a mechanism for the enhancement of LTM for individual episodes but also for cross-episode integration by enabling rapid behavioral generalization in the future. Integrating new episodes with past experiences is a key feature of our schema paradigm.

Our findings of a role for DA in modulating memory persistence are in line with the HPC-VTA loop model of Lisman and Grace (2005). When novelty is detected in the HPC, a neural signal is thought to pass first via the subiculum to the nucleus accumbens (NAcc). There, together with information from the prefrontal cortex, NAcc neurons stimulate the firing of the VTA. In keeping with this, Kentros et al. (2004) demonstrated that increased place field stability in CA1 is subject to neuromodulation by hippocampal DA released from terminals emanating from VTA neurons. They suggest that a higher-order cognitive process, such as attention, is mediated by one or more neuromodulatory inputs that switch short-term into long-term plasticity. In the presence of novelty, DA is released in the HPC, and 
early LTP is transformed into late LTP. Cellular consolidation of the memory representation of new PAs in our training paradigm would favor their integration into the schema via systems consolidation. Additional examination of this distinctive role of DA in HPC, compared with its error-correcting role in the striatum, would be to conduct tract-tracing studies of VTA projections to striatum and HPC and to test whether lesions specific to DA cells from the VTA projecting to the HPC have a selective impact on the persistence of memory but are without effect on initial encoding.

\section{References}

Adcock RA, Thangavel A, Whitfield-Gabrieli S, Knutson B, Gabrieli JD (2006) Reward-motivated learning: mesolimbic activation precedes memory formation. Neuron 50:507-517.

Bernabeu R, Bevilaqua L, Ardenghi P, Bromberg E, Schmitz P, Bianchin M, Izquierdo I, Medina JH (1997) Involvement of hippocampal cAMP/ cAMP-dependent protein kinase signaling pathways in a late memory consolidation phase of aversively motivated learning in rats. Proc Natl Acad Sci U S A 94:7041-7046.

Bliss TV, Collingridge GL (1993) A synaptic model of memory: long-term potentiation in the hippocampus. Nature 361:31-39.

Bontempi B, Laurent-Demir C, Destrade C, Jaffard R (1999) Timedependent reorganization of brain circuitry underlying long-term memory storage. Nature 400:671-675.

Buhot MC, Martin S, Segu L (2000) Role of serotonin in memory impairment. Ann Med 32:210-221.

Clayton NS, Dickinson A (1998) Episodic-like memory during cache recovery by scrub jays. Nature 395:272-274.

Dahlström A, Fuxe K (1964) Localization of monoamines in the lower brain stem. Experientia 20:398-399.

Day M, Langston R, Morris RGM (2003) Glutamate-receptor-mediated encoding and retrieval of paired-associate learning. Nature 424:205-209.

Dudai Y (2002) Memory from A to Z. Keywords, concepts and beyond. Oxford: Oxford UP.

Fink JS, Smith GP (1980) Mesolimbicocortical dopamine terminal fields are necessary for normal locomotor and investigatory exploration in rats. Brain Res 199:359-384.

Frey U, Morris RGM (1997) Synaptic tagging and long-term potentiation. Nature 385:533-536.

Frey U, Morris RGM (1998) Synaptic tagging: implications for late maintenance of hippocampal long-term potentiation. Trends Neurosci $21: 181-188$.

Frey U, Schroeder H, Matthies H (1990) Dopaminergic antagonists prevent long-term maintenance of posttetanic LTP in the CA1 region of rat hippocampal slices. Brain Res 522:69-75.

Frey U, Matthies H, Reymann KG, Matthies H (1991) The effect of dopaminergic D1 receptor blockade during tetanization on the expression of long-term potentiation in the rat CA1 region in vitro. Neurosci Lett 129:111-114

Gasbarri A, Verney C, Innocenzi R, Campana E, Pacitti C (1994) Mesolimbic dopaminergic neurons innervating the hippocampal formation in the rat: a combined retrograde tracing and immunohistochemical study. Brain Res 668:71-79.

Gasbarri A, Sulli A, Innocenzi R, Pacitti C, Brioni JD (1996) Spatial memory impairment induced by lesion of the mesohippocampal dopaminergic system in the rat. Neuroscience 74:1037-1044.

Gasbarri A, Sulli A, Packard MG (1997) The dopaminergic mesencephalic projections to the hippocampal formation in the rat. Prog Neuropsychopharmacol Biol Psychiatry 21:1-22.

Harvey JA (2003) Role of the serotonin 5- $\mathrm{HT}_{2 \mathrm{~A}}$ receptor in learning. Learn Mem 10:355-362.

Huang YY, Kandel ER (1995) D1/D5 receptor agonists induce a protein synthesis-dependent late potentiation in the CA1 region of the hippocampus. Proc Natl Acad Sci U S A 92:2446-2450.

Jay TM (2003) Dopamine: a potential substrate for synaptic plasticity and memory mechanisms. Prog Neurobiol 69:375-390.

Johnson A, Redish AD (2007) Neural ensembles in CA3 transiently encode paths forward of the animal at a decision point. J Neurosci 27:12176-12189.

Kentros CG, Agnihotri NT, Streater S, Hawkins RD, Kandel ER (2004) In- creased attention to spatial context increases both place field stability and spatial memory. Neuron 42:283-295.

Lazarov NE, Schmidt U, Wanner I, Pilgrim C (1998) Mapping of D1 dopamine receptor mRNA by non-radioactive in situ hybridization. Histochem Cell Biol 109:271-279.

Lisman JE, Grace AA (2005) The hippocampal-VTA loop: controlling the entry of information into long-term memory. Neuron 46:703-713.

Lisman JE, Otmakhova NA (2001) Storage, recall, and novelty detection of sequences by the hippocampus: elaborating on the SOCRATIC model to account for normal and aberrant effects of dopamine. Hippocampus 11:551-568.

Maaswinkel H, Whishaw IQ (1999) Homing with locale, taxon, and dead reckoning strategies by foraging rats: sensory hierarchy in spatial navigation. Behav Brain Res 99:143-152.

Martin SJ, Grimwood PD, Morris RGM (2000) Synaptic plasticity and memory: an evaluation of the hypothesis. Annu Rev Neurosci 23: 649-711.

Maviel T, Durkin TP, Menzaghi F, Bontempi B (2004) Sites of neocortical reorganization critical for remote spatial memory. Science 305:96-99.

Millan MJ, Newman-Tancredi A, Quentric Y, Cussac D (2001) The "selective" dopamine D1 receptor antagonist, $\mathrm{SCH} 23390$, is a potent and high efficacy agonist at cloned human serotonin2C receptors. Psychopharmacology (Berl) 156:58-62.

Moncada D, Viola H (2007) Induction of long-term memory by exposure to novelty requires protein synthesis: evidence for a behavioral tagging. J Neurosci 27:7476-7481.

Morris RGM (2006) Elements of a neurobiological theory of hippocampal function: the role of synaptic plasticity, synaptic tagging and schemas. Eur J Neurosci 23:2829-2846.

O'Carroll CM, Morris RGM (2004) Heterosynaptic co-activation of glutamatergic and dopaminergic afferents is required to induce persistent long-term potentiation. Neuropharmacology 47:324-332.

O'Carroll CM, Martin SJ, Sandin J, Frenguelli B, Morris RGM (2006) Dopaminergic modulation of the persistence of one-trial hippocampusdependent memory. Learn Mem 13:760-769.

Overton DA (1964) State-dependent or "dissociated" learning produced with pentobarbital. J Comp Physiol Psychol 57:3-12.

Packard MG, White NM (1991) Dissociation of hippocampus and caudate nucleus memory systems by posttraining intracerebral injection of dopamine agonists. Behav Neurosci 105:295-306.

Paxinos G, Watson C (1998) The rat brain in stereotaxic coordinates. San Diego: Academic.

Rossato JI, Bevilaqua LR, Izquierdo I, Medina JH, Cammarota M (2009) Dopamine controls persistence of long-term memory storage. Science 325:1017-1020.

Rudy JW, Sutherland RJ (2008) Is it systems or cellular consolidation? Time will tell. An alternative interpretation of the Morris group's recent science paper. Neurobiol Learn Mem 89:366-369.

Schott BH, Sellner DB, Lauer CJ, Habib R, Frey JU, Guderian S, Heinze HJ, Düzel E (2004) Activation of midbrain structures by associative novelty and the formation of explicit memory in humans. Learn Mem 11:383-387.

Schultz W (2007) Behavioral dopamine signals. Trends Neurosci 30:203-210.

Schultz W, Dickinson A (2000) Neuronal coding of prediction errors. Annu Rev Neurosci 23:473-500.

Schultz W, Apicella P, Scarnati E, Ljungberg T (1992) Neuronal activity in monkey ventral striatum related to the expectation of reward. J Neurosci 12:4595-4610.

Shohamy D, Wagner AD (2008) Integrating memories in the human brain: hippocampal-midbrain encoding of overlapping events. Neuron 60:378-389.

Smith-Roe SL, Kelley AE (2000) Coincident activation of NMDA and dopamine $D_{1}$ receptors within the nucleus accumbens core is required for appetitive instrumental learning. J Neurosci 20:7737-7742.

Steele RJ, Morris RGM (1999) Delay-dependent impairment of a matchingto-place task with chronic and intrahippocampal infusion of the NMDAantagonist D-AP5. Hippocampus 9:118-136.

Steinfels GF, Heym J, Strecker RE, Jacobs BL (1983) Behavioral correlates of dopaminergic unit activity in freely moving cats. Brain Res 258:217-228. Swanson LW (1982) The projections of the ventral tegmental area and ad- 
jacent regions: a combined fluorescent retrograde tracer and immunofluorescence study in the rat. Brain Res Bull 9:321-353.

Swanson-Park JL, Coussens CM, Mason-Parker SE, Raymond CR, Hargreaves EL, Dragunow M, Cohen AS, Abraham WC (1999) A double dissociation within the hippocampus of dopamine D1/D5 receptor and beta-adrenergic receptor contributions to the persistence of longterm potentiation. Neuroscience 92:485-497.

Taghzouti K, Simon H, Louilot A, Herman JP, Le Moal M (1985) Behavioral study after local injection of 6-hydroxydopamine into the nucleus accumbens in the rat. Brain Res 344:9-20.

Tse D, Langston RF, Kakeyama M, Bethus I, Spooner PA, Wood ER, Witter MP, Morris RGM (2007) Schemas and memory consolidation. Science 316:76-82.

Tse D, Langston RF, Bethus I, Wood ER, Witter MP, Morris RGM (2008)
Does assimilation into schemas involve systems or cellular consolidation? It's not just time. Neurobiol Learn Mem 89:361-365.

Umegaki H, Munoz J, Meyer RC, Spangler EL, Yoshimura J, Ikari H, Iguchi A, Ingram DK (2001) Involvement of dopamine $\mathrm{D}_{2}$ receptors in complex maze learning and acetylcholine release in ventral hippocampus of rats. Neuroscience 103:27-33.

Valdes JL, McNaughton BL, Fellous JM (2008) Reactivation of populations of ventral tegmental area neurons in the rat. Soc Neurosci Abstr 34:687.19.

Willuhn I, Steiner H (2006) Motor-skill learning-associated gene regulation in the striatum: effects of cocaine. Neuropsychopharmacology 31:2669-2682.

Wittmann BC, Schott BH, Guderian S, Frey JU, Heinze HJ, Düzel E (2005) Reward-related FMRI activation of dopaminergic midbrain is associated with enhanced hippocampus-dependent long-term memory formation. Neuron 45:459-467. 